\title{
Echocardiographic evaluation of mechanical dyssynchrony in heart failure patients with reduced ejection fraction
}

\author{
Zsolt Gyalai $^{\mathrm{a}, *}$, Zsuzsanna Jeremiás ${ }^{\mathrm{a}}$, Emoke Baricz ${ }^{\mathrm{a}}$, Roxana Rudzik ${ }^{\mathrm{b}}$ and Dan Dobreanu ${ }^{\mathrm{b}}$ \\ ${ }^{a}$ Department of Cardiology, University of Medicine and Pharmacy Târgu Mureş, Mureş County \\ Clinical Hospital, Târgu Mureş, Romania \\ ${ }^{\mathrm{b}}$ Department of Cardiology, University of Medicine and Pharmacy Târgu Mureş, Institute of \\ Cardiovascular Diseases and Transplant, Târgu Mureş, Romania
}

\begin{abstract}
The aim of this study is to observe the differences between mechanical and electrical dyssynchrony in patients with impaired systolic ventricular function and symptomatic heart failure and to highlight the importance of mechanical dyssynchrony besides electrical dyssynchrony in clinical guidelines and clinical practice. Fifty-eight patients with heart failure, who are with the New York Heart Association (NYHA) functional class II-IV and left ventricular ejection fraction (LVEF) under $35 \%$, were enrolled. Patients were divided into two groups, according to the duration of QRS complex ( $>120 \mathrm{~ms}$ and $\leqslant 120 \mathrm{~ms}$ respectively). Echocardiographic parameters of interventricular (interventricular mechanical delay - IMD) and intraventricular (septal-to-posterior wall motion delay - SPWMD) dyssynchrony were measured in both groups. Results indicate that the duration of the QRS complex (i.e. electrical dyssynchrony) is not a fully reliable indicator of ventricular dyssynchrony; therefore ecocardiographic evaluation of mechanical dyssynchrony should also be recommended for better selection of candidates for cardiac resynchronization therapy (CRT).
\end{abstract}

Keywords: Ventricular dyssynchrony, heart failure, echocardiography, cardiac resynchronization therapy (CRT)

\section{Introduction}

Ventricular dyssynchrony appears by altering of the electrical activation sequence of the heart or by myocardial ischemia [1]. Thus, left bundle branch block (LBBB) reflects a delay in the left ventricular systole and diastole compared with the electrical activation of the right ventricle. Early septal contraction leads to a dyssynchrone outward motion of the left ventricular lateral wall, so instead of contributing to the ejection of blood from the left ventricle, it acts as a ventricular reservoir. This contractile dyssynchrony of one or more segments of the left ventricle leads to mechanical dyssynchrony [1,2].

Multiple studies have proven the existence of statistically significant correlation between the presence of ventricular (mechanical) dyssynchrony and the prolonged duration of QRS complex (electrical dyssynchrony) $[1,3]$.

\footnotetext{
${ }^{*}$ Corresponding author: Zsolt Gyalai, Department of Cardiology, University of Medicine and Pharmacy Târgu Mureş, Mureş County Clinical Hospital, str. Gh. Marinescu, nr. 1, RO-540103, Târgu Mureş, Romania. Tel.: +40 745 059149; E-mail: zsolt.gyalai@umftgm.ro.
} 
According to the European Society of Cardiology's 2013 guidelines [4], cardiac resynchronization therapy (CRT) has class I indication in patients with sinus rhythm in NYHA functional class II, III and ambulatory class IV heart failure, QRS duration $>120 \mathrm{~ms}$ and a persistently reduced left ventricular ejection fraction (LVEF $<35 \%$ ), with optimal pharmacological therapy. CRT has class IIa indication in patients with atrial fibrillation and symptomatic heart failure (HF) (NYHA functional class III and ambulatory class IV), QRS duration $>120 \mathrm{~ms}$ and left ventricular ejection fraction (LVEF) $<35 \%$, despite optimal pharmacological therapy. These guidelines state that echocardiographic evaluation should be used only for assesment of left ventricular systolic function, without any other contribution to determining the presence of mechanical dyssynchrony, the only dyssynchrony parameter used in establishing the indication for CRT being the duration of the QRS complex (electrical dyssynchrony).

Despite the existence of a significant correlation between mechanical and electrical dyssynchrony, it is widely known that these two types of dyssynchrony are quite different $[5,6]$. Hence there are a number of reasons why echocardiographic assessment of mechanical dyssynchrony may be an important, yet easy, reliable and cost-effective parameter to measure in addition to electrical dyssynchrony and can be of value in clinical decisions regarding treatment of patients with HF, such as better selection of patients for CRT (given the fact that QRS duration has not entirely predicted response to CRT), lead placement, assessing response to CRT and optimizing pacemaker settings [5].

\section{Materials and methods}

The aim of this study was to evaluate and compare the parameters of interventricular and intraventricular dyssynchrony (markers of mechanical dyssynchrony) in patients with wide (electrically dyssynchrone) and narrow QRS complexes, in order to establish how parameters of electrical dyssynchrony (i.e. wide QRS on surface ECG) correlate with those of mechanical (contractile) dyssynchrony.

The study was approved by the hospital's ethics committee, and written informed consent was obtained from each of the enrolled patients.

Fifty-eight patients with heart failure NYHA functional class II-IV and left ventricular ejection fraction under 35\% were enrolled in a comparative, prospective study (authors own cases). All of the patients were admitted to the Cardiology Department of Internal Medicine Clinic IV - Târgu-Mureş, between January 2010-December 2013, for specific treatment of heart failure.

Patients were divided into two groups based on the duration of QRS complex: one group with wide $(>120 \mathrm{~ms})$ and another with narrow $(\leqslant 120 \mathrm{~ms})$ QRS complexes. Age, sex, NYHA functional class, electrocardiographic (ECG) and echocardiographic parameters were registered in both groups. ECG parameters included the presence of sinus rhythm or atrial fibrillation and duration of QRS complex. Left ventricular end diastolic diameter (LVEDD), left ventricle ejection fraction (using Simpson's biplane method) and markers of mechanical dyssynchrony were assesed by echocardiography.

Echocardiographic evaluation and measurements were performed with an ALOKA Prosound $\alpha 10$ device, by the same examiner in each of the cases to reduce inter-examiner variations. Two dyssynchrony parameters were determined:

- Interventricular mechanical delay (IMD) for interventricular dyssynchrony - quantifiable as the time difference between preejection period for the left and right ventricles, measured by CW EchoDoppler method (IMD = left ventricular preejection period - right ventricular preejection period).

- Septal-to-posterior wall motion delay (SPWMD) for intraventricular dyssynchrony, measured in parasternal short axis sections at the level of papillary muscles in 2D guided - "M" mode. 
Table 1

Basic clinical characteristics of the two groups

\begin{tabular}{lll}
\hline & Narrow QRS $(\leqslant 120 \mathrm{~ms})$ & Wide QRS $(>120 \mathrm{~ms})$ \\
\hline Number of patients & 16 & 42 \\
Male & $81.25 \%$ & $88.09 \%$ \\
Mean age \pm SD (years) & $44.17 \pm 13.72$ & $72.00 \pm 9.17$ \\
Sinus rhythm & $81.25 \%$ & $61.90 \%$ \\
Atrial fibrillation & $18.75 \%$ & $38.09 \%$ \\
Mean NYHA class & 2.83 & 2.88 \\
Mean LVEDD \pm SD $(\mathrm{mm})$ & $65.83 \pm 6.97$ & $60.31 \pm 10.36$ \\
Mean LVEF \pm SD $(\%)$ & $18.45 \pm 9.8$ & $21.08 \pm 8.99$ \\
\hline
\end{tabular}

Table 2

The prevalence of interventricular dyssynchrony (nr. of patients)

\begin{tabular}{lcc}
\hline & Narrow QRS & Wide QRS \\
\hline IMD $<40 \mathrm{~ms}$ & 11 & 20 \\
IMD $>40 \mathrm{~ms}$ & 5 & 22 \\
\hline
\end{tabular}

Table 3

The prevalence of intraventricular dyssynchrony (nr. of patients)

\begin{tabular}{lcc}
\hline & Narrow QRS & Wide QRS \\
\hline SPWMD $<130 \mathrm{~ms}$ & 10 & 5 \\
SPWMD $>130 \mathrm{~ms}$ & 6 & 37 \\
\hline
\end{tabular}

Statistical data analysis: based on the presence/absence of electrical dyssynchrony, values of both mechanical dyssynchrony parameters were compared. Calculations were performed using Microsoft Office Excel 2003 and GraphPad InStat 3.06, applying Kolmogorov-Smirnov's goodness-of-fit test, Fischer's test for variance and homoscedatic Student's unpaired t-test for data regarding IMD, respectively, MannWhitney test for SPWMD.

\section{Results}

Patients were predominantly male $(86.2 \%)$, with an average age of 64.4 years. The main underlying cause for cardiomyopathy was ischemia (75.86\%). Sinus rhythm was present in $67.24 \%$ of the cases, while $32.75 \%$ of patients were in atrial fibrillation. In $72.41 \%$ of the cases, underlying cardiomyopathy was in dilated stage (left ventricular end diastolic diameter - LVEDD > $55 \mathrm{~mm}$ ), the mean LVEDD was $61.81 \mathrm{~mm}$. Based on the LVEF, all of the enrolled patients had left ventricular disfunction, with a mean value of $20.2 \%$. Forty-two patients were identified with prolonged ventricular depolarization (QRS > $120 \mathrm{~ms}$ ) on the surface ECG performed at the time of admission to hospital (main characteristics of the two groups are shown in Table 1). Additionally, $27.58 \%$ of the patients had permanent ventricular stimulation with the lead placed in the right ventricle. According to distribution of NYHA functional class, $27.58 \%$ of patients were NYHA class II, $58.62 \%$ in NYHA class III and $13.79 \%$ in NYHA class IV respectively.

Overall, $46.55 \%$ of patients had positive criteria for interventricular dyssynchrony (IMD $>40 \mathrm{~ms}$ ). In the wide QRS group, interventricular dysynchrony criteria were present in $52.38 \%$ of patients, while $47.61 \%$ had preserved interventricular synchronism (IMD $<40 \mathrm{~ms}$ ). In the narrow QRS group, $31.25 \%$ of patients had interventricular dyssynchrony, while in $68.75 \%$ of the group no dyssynchrony parameters were detectable (see Table 2 and Fig. 1). Comparative analysis of IMD values between the two groups (wide QRS versus narrow QRS complexes) showed that there was no statistically significant difference between IMD values of the two groups $(p=0.222)$.

Overall, $74.13 \%$ of patients had positive criteria for intraventricular dyssynchrony (SPWMD > $130 \mathrm{~ms}$ ). In the wide QRS group, 88.09\% of patients had intraventricular dyssynchrony, while $11.90 \%$ 


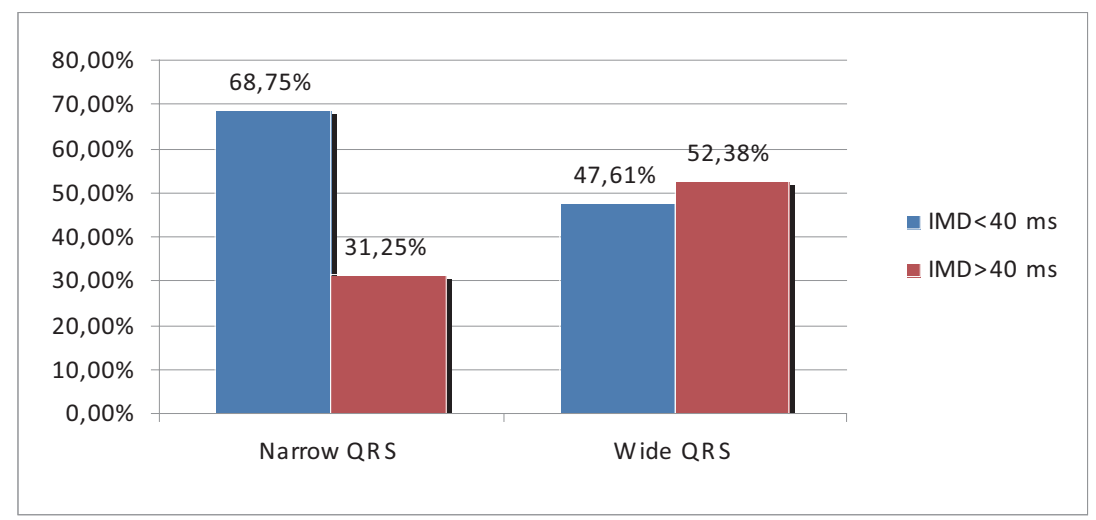

Fig. 1. Prevalence of interventricular dyssynchrony in narrow QRS vs. wide QRS group.

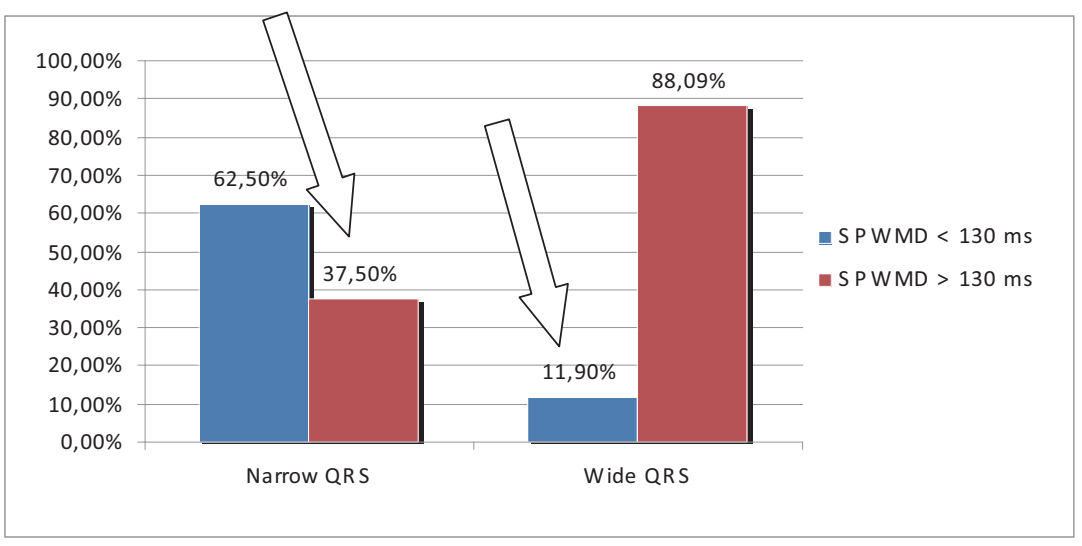

Fig. 2. Prevalence of intraventricular dyssynchrony in narrow QRS vs. wide QRS group.

had preserved intraventricular synchrony (SPWMD $<130 \mathrm{~ms}$ ). In the narrow QRS group, 37.5\% of patients were found to have intraventricular dyssynchrony, while $62.5 \%$ had normal intraventricular synchrony (see Table 3 and Fig. 2). Comparative analysis of SPWMD values between the two groups (wide vs. narrow QRS complexes) showed that there was a statistically significant difference between SPWMD values of the two groups $(p=0.027)$.

\section{Discussion}

There is a statistically significant association between the studied intraventricular dyssynchrony parameter (SPWMD) and duration of the QRS complex ( $p=0.027)$, but not between interventricular dyssynchrony determined by IMD and duration of QRS complex $(p=0.222)$. These data are comparable to multiple similar studies $[1,3,5,6]$. Results show that out of the two parameters, SPWMD is the more accurate and more reliable parameter to assess mechanical dyssynchrony.

Most patients (88\%) with heart failure NYHA class II-IV, LVEF $<35 \%$ and wide QRS have intraventricular dyssynchrony (SPWMD $>130 \mathrm{~ms}$ ), but $11.9 \%$ of them don't have intraventricular dyssynchrony (see Fig. 2) and an even greater proportion (47.6\%) don't have interventricular dyssynchrony (see Fig. 1), 
thus they could represent the group that would not benefit from CRT. These patients will most probably represent the majority of non-responders to CRT. Similar results have been obtained in other studies as well, where about $20 \%$ of patients with $\mathrm{EF}<35 \%$ and duration of QRS $>150 \mathrm{~ms}$ did not meet the criteria of LV mechanical dyssynchrony $[7,8]$. Parameters of mechanical dyssynchrony determined by echocardiography should be used as additional criteria in the evaluation of candidates for cardiac resynchronisation therapy, alongside current ECG criteria, because the echocardiographic method is a simple, reliable and reproductible method, which can be useful in detection of possible non-responders to CRT. Still, it is difficult to predict the group of non-responders and it may be neccesary to consider other parameters as well, like the etiology of the cardiomyopathy, QRS morphology and other possible factors, beside echocardiographic and ECG criteria $[9,10]$.

Another important issue remains the fact that using QRS duration as selection criteria excludes a number of patients with narrow QRS (without electrical dyssynchrony), who could still benefit from CRT. In this study, an important percentage (about 35\%) of the narrow QRS group with heart failure NYHA class II-IV and LVEF $<35 \%$ have mechanical dyssynchrony $(37.5 \%$ and $31.25 \%$ considering SPWMD and IMD respectively). Other studies have shown similar results [5,6,11-14], using different evaluation techniques, approximately 50\% of patients with heart failure and duration of QRS complex $<120 \mathrm{~ms}$, meet the criteria for mechanical dyssynchrony $[8,15]$ and these patients show clinical improvement after CRT in some studies [16]. In another randomized, multicentric trial, CRT does not reduce the rate of death or hospitalization for heart failure and even may increase mortality in these patients [17].

\section{Conclusion}

- There is a statistically significant association between electrical dyssynchrony (QRS duration) and intraventricular dyssynchrony, but not between electrical and interventricular dyssynchrony.

- There is a group of patients with narrow QRS (without electrical dyssynchrony), who present important mechanical dyssynchrony at echocardiographic examination. These patients might represent a target group for CRT. Thereby, using the duration of QRS complexes as the only selection criteria for cardiac dyssynchrony in CRT candidates, without evaluation of mechanical (ultrasound) criteria, excludes a number of patients with narrow QRS who still might benefit from CRT in selected cases.

- The group of non-responders to CRT might consist of those patients who have evidence of electrical dissynchrony (large QRS), but not of mechanical dyssynchrony.

- The duration of QRS complexes alone seems to be an insensitive indicator of ventricular dyssynchrony, hence ultrasound evaluation should also be recommended for better selection of possible CRT candidates.

\section{Acknowledgements}

This paper is partly supported by the Sectorial Operational Programme Human Resources Development (SOP HRD), financed by the European Social Fund and by the Romanian Government under the contract number POSDRU/CPP107/DMI1.5/80641. 


\section{References}

[1] W. T. Abraham, Devices for Monitoring and Managing Heart Failure, in: R. Bonow, D. Mann, D. Zipes, P. Libby (eds): Braunwald's Heart Disease. A textbook of cardiovascular medicine, $9^{\text {th }}$ edition. Ed. Elsevier Saunders, 2011, 578-585.

[2] D. A. Kass, Ventricular resynchronization: pathophophysiology and identification of responders, Rev Cardiovasc Med 4 (suppl 2) (2003), S3-13.

[3] D. Farwell, N. R. Patel, A. Hall et al., How many people with heart failure are appropriate for biventricular resynchronization? European Heart Journal 21 (2000), 1246.

[4] M. Brignole, A. Auricchio et al., 2013 ESC Guidelines on cardiac pacing and cardiac resynchronization therapy, European Heart Journal 34 (2013), 2281-2329.

[5] J. Bank, V. Burn and M. Gage, Echocardiographic measurement of mechanical dyssynchrony in heart failure and cardiac resynchronization therapy, US Cardiology 7(1) (2010), 24-32.

[6] K. Han-Na, H. Hideyuki, T. Hidekazu, O. Olusequn, S. David, G. John, Comparison of dyssynchrony in heart failure patients with narrow QRS to those with wide QRS: Implications for resynchronization therapy, Circulation 118 (2008), 869-870.

[7] J. Newton, J. Davies, J. Kovac et al., Prevalence of echocardiographic left ventricular contractile dyssynchrony in patients with left bundle branch block: Impact on pacing, J Am Coll Cardiol 43 (2004), 130A.

[8] R. O. Jurcut, B. A. Popescu, R. Ciudin et al., Is QRS width sufficient in selecting patients with intraventricular asynchronism in dilated cardiomyopathy? Echocardiographical study, EUROECHO 9, Eur J Echocard 6, suppl 1 (2005).

[9] E. S. Chung, A. R. Leon, L. Tavazzi et al., Results of the predictors of response to CRT (PROSPECT) trial, Circulation 117(20) (2008), 2567-2569.

[10] A. Auricchio, F. W. Prinzen, Non-responders to cardiac resynchronization therapy: The magnitude of the problem and the issues, Circ J. 75 (2011), 521-527.

[11] Y. G. Sun, W. F. Shen, F. R. Zhang, Y. Q. Xu, W. Ruan, Q. H. Zhao, Left ventricular dyssynchrony evaluated by echocardiography in chronic heart failure patients with normal and wide QRS duration, Zhonghua Xin Xue Guan Bing Za Zhi 36(1) (Jan 2008), 44-48.

[12] G. B. Bleeker, M. J. Schalij, S. G. Molhoek et al., Relationship between QRS duration and left ventricular dyssynchrony in patients with end-stage heart failure, J Cardiovasc Electrophysiol 15 (2004), 544-549.

[13] S. Kapetanakis, M. T. Kearney, A. Siva, N. Gall, M. Cooklin, M. J. Monaghan, Real-time three-dimensional echocardiography, a novel technique to quantify global left ventricular mechanical dyssynchrony, Circulation 112 (2005), 992-1000.

[14] U. Narasimha, QRS duration is an insensitive marker of left ventricular dyssynchrony in patients with end-stage heart failure, Medscape, 27 May 2004.

[15] C. M. Yu, H. Lin, Q. Zhang et al., High prevalence of left ventricular systolic and diastolic asynchrony in patients with congestive heart failure and normal QRS duration, Heart 89 (2003), 54-60.

[16] M. Gasparini, M. Mantica, P. Galimberti et al., Beneficial effects of biventricular pacing in patients with a "narrow" QRS, Pacing Clin Electrophysiol 26 (2003), 169-174.

[17] F. Ruschitzka, W. T. Abraham, J. P. Singh, J. J. Bax et al., Cardiac-resynchronization therapy in heart failure with a narrow QRS complex, N Engl J Med 369 (2013), 1395-1405. 